Western Washington University

Western CEDAR

5-10-1993

\title{
Paleomagnetic Evidence of Vertical Axis Block Rotations from the Mesozoic of Northern Chile
}

Paul D. Riley

Myrl E. BeckJr.

Western Washington University, myrl.beck@wwu.edu

Russ R. Burmester

Western Washington University, russ.burmester@wwu.edu

Constantino Mpodozis

Alfredo Garcia

Follow this and additional works at: https://cedar.wwu.edu/geology_facpubs

Part of the Geology Commons

\section{Recommended Citation}

Riley, Paul D.; Beck Jr., Myrl E.; Burmester, Russ R.; Mpodozis, Constantino; and Garcia, Alfredo, "Paleomagnetic Evidence of Vertical Axis Block Rotations from the Mesozoic of Northern Chile" (1993). Geology Faculty Publications. 30.

https://cedar.wwu.edu/geology_facpubs/30 


\section{PALEOMAGNETIC EVIDENCE OF VERTICAL AXIS BLOCK ROTATIONS} FROM THE MESOZOIC OF NORTHERN CHILE

Paul D. Riley, Myrl E. Beck, Jr., and Russell F. Burmester

Department of Geology, Western Washington University, Bellingham

Constantino Mpodozis and Alfredo Garcia ${ }^{1}$

Servicio Nacional de Geologia y Mineria, Chile, Santiago

\begin{abstract}
We present paleomagnetic results for three Mesozoic formations from northern Chile: La Ternera Formation (Upper Triassic), Quebrada Monardes Formation (Upper Jurassic); Cerrillos Formation (Upper Cretaceous). Results from the Cerrillos are divided into eastern (Cuesta El GaO (CEG)) and western (Elisa De Bordo (EBD)) localities. Most specimens from La Ternera volcanic and sedimentary rocks are magnetically stable, as shown by alternating field and thermal demagnetization. More complicated but still reliable results were obtained from Quebrada Monardes red beds. Normal and reverse polarities are present in both units; means of both populations are antiparallel at 958 confidence. The Quebrada Monardes. Formation also yields positive conglomerate and fold tests. Paleomagnetic poles for La Ternera and Quebrada Monardes are $60.9 \mathrm{~S}, 218.3 \mathrm{E}\left(\mathrm{A} 95,7.8^{\circ}\right)$, and $66.9 \mathrm{~S}, 191.6 \mathrm{E}\left(\mathrm{A95}, 12.7^{\circ}\right)$, respectively. Comparison with appropriate reference poles shows that this region of Chile has undergone about $25^{\circ}$ of clockwise rotation, with negligible latitudinal transport. Cerrillos CEG results are less reliable and possibly complicated by remagnetization during emplacement of early Tertiary intrusives. Most Cerrillos EDB specimens are stable, but marked increase in scatter upon unfolding suggests remagnetization. Results for the Cerrillos CEG locality, which is contiguous to the sampling area of the La Ternera and Quebrada Monardes formations, show only about half the rotation of those two units, suggesting that rotation commenced after deposition of the Quebrada Monardes rocks in the Late Jurassic and was approximately half complete by the time cerrillos CEG rocks acquired their magnetization. Cerrillos EDB results come from an area approximately $40 \mathrm{~km}$ to the west; these show roughly $45^{\circ}$ of clockwise rotation. Dispersion is very low between EDB sites, suggesting that secular variation may not be completely averaged. Nevertheless, the great difference in direction between the two cerrillos localities suggests that they lie in different structural blocks.
\end{abstract}

\section{Introduction}

Paleomagnetic studies provide an additional dimension to the tectonic analysis of mountain belts. To cite a well-known example, paleomagnetic evidence from the North American Cordillera shows that crustal blocks located on the western edge of the continent have experienced variable amounts of vertical axis rotation, in most cases accompanied by displacement parallel to the continental margin [Beck, 1976; Irving et al., 1980; stone et al., 1982 ]. For the south America Cordillera the pattern is quite differ-

1 Now at Geoestudios, Limitada, Santiago, Chile.

Copyright 1993 by the American Geophysical Union.

Paper number $92 \mathrm{JB} 02691$.

0148-0227/93/92JB-0269i\$05.00 ent; data show little or no evidence of terrane accretion or large-scale block displacement since the Paleozoic [Ramos et al., 1986; Beck, 1988]. However, there is an interesting pattern of rotation; paleomagnetic studies demonstrate that rocks north of the Peru/Chile border are rotated counterclockwise, whereas in nearly all studies the rocks from chile show clockwise rotation. Broadly speaking, two models have been advanced to explain these discordant paleomagnetic declinations, both of which involve interaction between the subducted Nazca and the overriding South American plates.

The orocline. Carey [1954] proposed that the abrupt deflection in Andean tectonic and topographic trends near Arica (northern Chile) represents oroclinal bending (Figure la). Although originally dismissed by many scientists as unlikely, this idea has been shown to fit paleomagnetic results from Peru [e.g., Kono et al., 1989] and Isacks [1988] has proposed a highly plausible model to account for oroclinal bending in the context of Andean subduction. Small-block rotation models. Margin-parallel shear caused by oblique subduction also can explain the different sense of rotation (Beck.

1987, 1988]. In models of this sort (Figure 1b), crustal blocks rotate in a shear zone caused by oblique convergence between the Nazca and South American plates; the sense of rotation is controlled by the sense of obliquity which reverses at the Arica deflection where the trend of the plate margin changes abruptly. This mechanism might be expected to drive rotations that decrease with increasing distance from the trench [Beck et al,. 1986; England and Wells, 1991], although rotations could be located far inland of the trench along major strike slip faults. Local geological conditions should control the size of the individual blocks and also might influence the timing and amount of rotation.

other kinematic models relate in situ block rotations to crustal extension within the Andean forearc. For instance, Hartley et al. [1988] suggest that rotations occur during roll-back of the subducted slab (Figure ic). A related model is described by Irwin et al. [1987]. These models also predict that the amount of rotation should decrease with distance from the trench. If models of this sort are correct, then the amount of rotation might vary from block to block but ought to be related in some fairly obvious way to plutons or extensional structures. A weakness of this class of model is the inability to account for a consistent sense of rotation and its relationship to the Arica deflection. Presumably, the sense of obliquity in convergence must be involved.

The paleomagnetic study described in this report includes results from Upper Triassic, Upper Jurassic, and Upper Cretaceous rock units, distributed over a wide geographic area (Figure $2)$. We originally hoped that results from these rocks could be used to constrain the age and duration of rotation, if any was found. We deliberately sampled over a large geographical area in order to detect and isolate independ- 

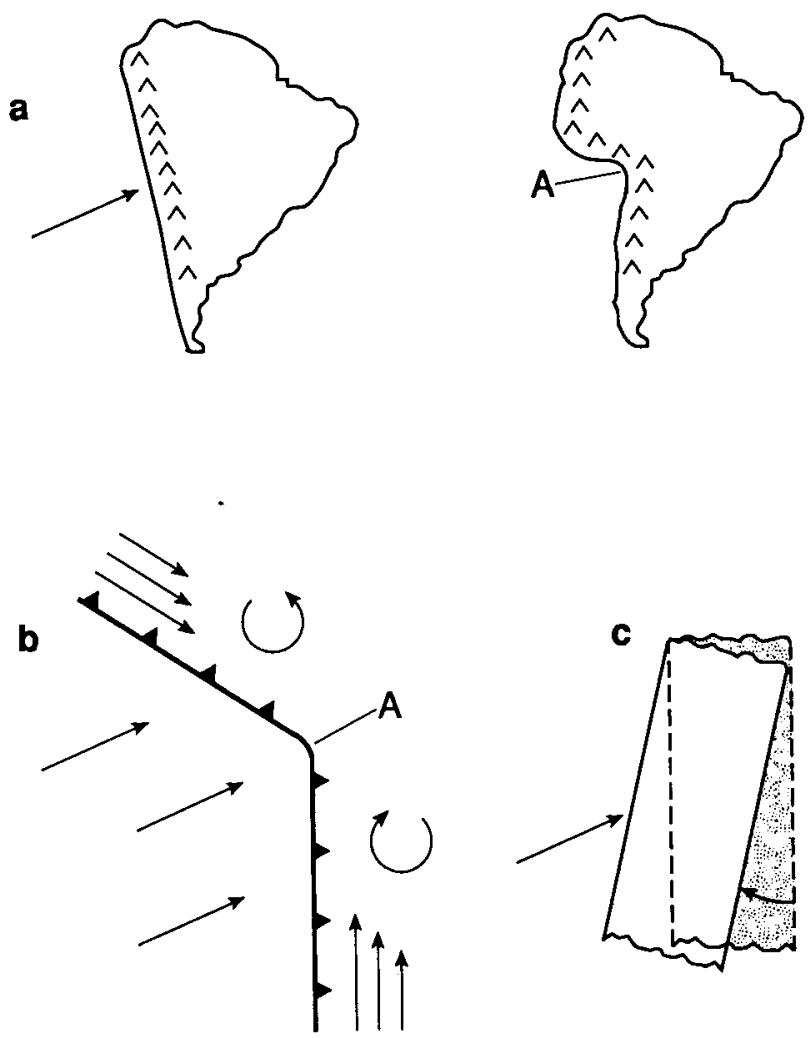

Fig. 1. Several tectonic models proposed to account for vertical axis rotations of crustal blocks in western South America. (a) Orocline. Interaction of South America with the Nazca plate causes differential crustal thickening and indentation of the continental margin; oroclinal bending is one result. A is Arica. (b) Small block rotation driven by oblique convergence. Subduction of the Nazca plate beneath an inherited bend in the South American coastline causes margin-parallel shear and block rotations.

Sense of shear and rotation changes at Arica (A). (C) Rotation by differential extension. Greater extension in the South than in the North produces vertical axis block rotation. Area of extension is shown.

ently rotated blocks, and perhaps shed some light on the rotation mechanism.

\section{Geology of the Sampling Area}

The study area is located on the southern Altiplano plateau just west of the Andean divide (Figure 2). Uplift along north-south oriented faults exposes Paleozoic basement, overlain by a thick sequence of Mesozoic volcanic and sedimentary rocks. Structure in the area is relatively simple; most layered rocks are subhorizontal, except locally where bedding is affected by faults. Paleocene and Eocene plutonic rocks locally intrude the Mesozoic section.

our sampling included Triassic volcanic and sedimentary rocks (La Ternera Formation), Upper Jurassic terrigenous red beds (Quebrada Monardes Formation), and Cretaceous volcaniclastic and volcanic rocks (Cerrillos Formation).

Upper Triassic La Ternera Formation in the sampling area consists of intermediate to felsic lava flows and pyroclastic rocks. According to Sepulveda and Naranjo [1982], La Ternera Formation in nearby areas contains a sedimentary member from which Triassic plant fossils have been collected. The La Ternera Formation unconformably overlies Paleozoic granitic rocks and is overlain conformably by the abundantly fossiliferous marine Lower Jurassic Lautaro Limestone, which we did not sample.

Upper Jurassic red beds of the Quebrada Monardes Formation conformably overlie the Lautaro Limestone. The Quebrada Monardes Formation is a sequence of terrigenous sedimentary rocks deposited in an arid to semi-arid environment [Bell, 1982 ]. Sedimentary facies reflect deposition of aeolian dunes, alluvial fans, braided streams, playa mudflats, saline lakes, and coastal lagoons. Andesitic sills and some flows also are present within this unit. The upper Jurassic age is constrained by its stratigraphic position and by relatively scarce paleobotanical material [Sepulveda and Naranjo, 1982].

Cretaceous volcanic and volcaniclastic rocks of the Cerrillos Formation conformably overlie the Quebrada Monardes Formation. These consist of conglomerates, andesitic lavas and breccias, pyroclastic rocks, and scarce limestones. These Cretaceous rocks have been attributed to an "aborted marginal basin" (Mpodozis and Ramos, 1990). The age of the Cerrillos Formation is poorly known. West of Copiapo (western margin of Figure 2), the Cerrillos Formation discordantly overlies rocks of Barremian age. Whole rock and hornblende K/Ar ages on Cerrillos volcanics are Paleocene-Eocene (Zentilli, 1974). but in view of the number of early Tertiary intrusions known in the area these ages may be partially reset.

\section{Paleomagnetic Sampling, Measurements, and} Statistical Techniques

In two field seasons we collected oriented samples (usually 6-8) from 127 sites, disproportionately from the well-exposed Quebrada Monardes Formation. In this paper we present all our results for the La Ternera and cerrillos formations, together with preliminary results for the Quebrada Monardes Formation. As discussed below, Quebrada Monardes red beds tend to have complicated magnetizations that are difficult to resolve.

Most samples were drilled and oriented in the field, using a combination solar-magnetic compass. At some sites we collected oriented hand samples, owing to the remote location of the outcrop or to equipment failure. Specimens were measured on a Schonstedt spinner magnetometer and progressively demagnetized within magnetic shields using either a schonstedt alternating field (AF) tumbling specimen demagnetizer or a home-built open-air furnace. Pilot specimens from nearly all sites have been analyzed to help us choose the most efficient demagnetization techniques. Here we report final results for all acceptable sites completed to date.

Magnetic component analysis was performed by least squares fitting of free lines to straightline segments selected from orthogonal diagrams (Kirschvink, 1980). Site mean directions were computed by combining sample directions using Fisher [1953] statistics. Sites with mean directions that were poorly determined (radius of circle of 958 confidence $>20^{\circ}$ ) were eliminated. Virtual geomagnetic poles (VGP) were calculated by averaging site mean VGPs. Relevant site statistics are given in Tables $1-3$; details of lithology, location, and demagnetization procedure are summarized in Table 4.

\section{Paleomagnetic Results}

Behavior of representative samples during demagnetization is shown in Figure 3 , and the cluster of site mean directions for each formation is illustrated in Figures 4-7. "Expected directions," also shown in Figures 4-7, are geomagnetic field directions calculated from the appropriate reference poles for the South Ameri- 

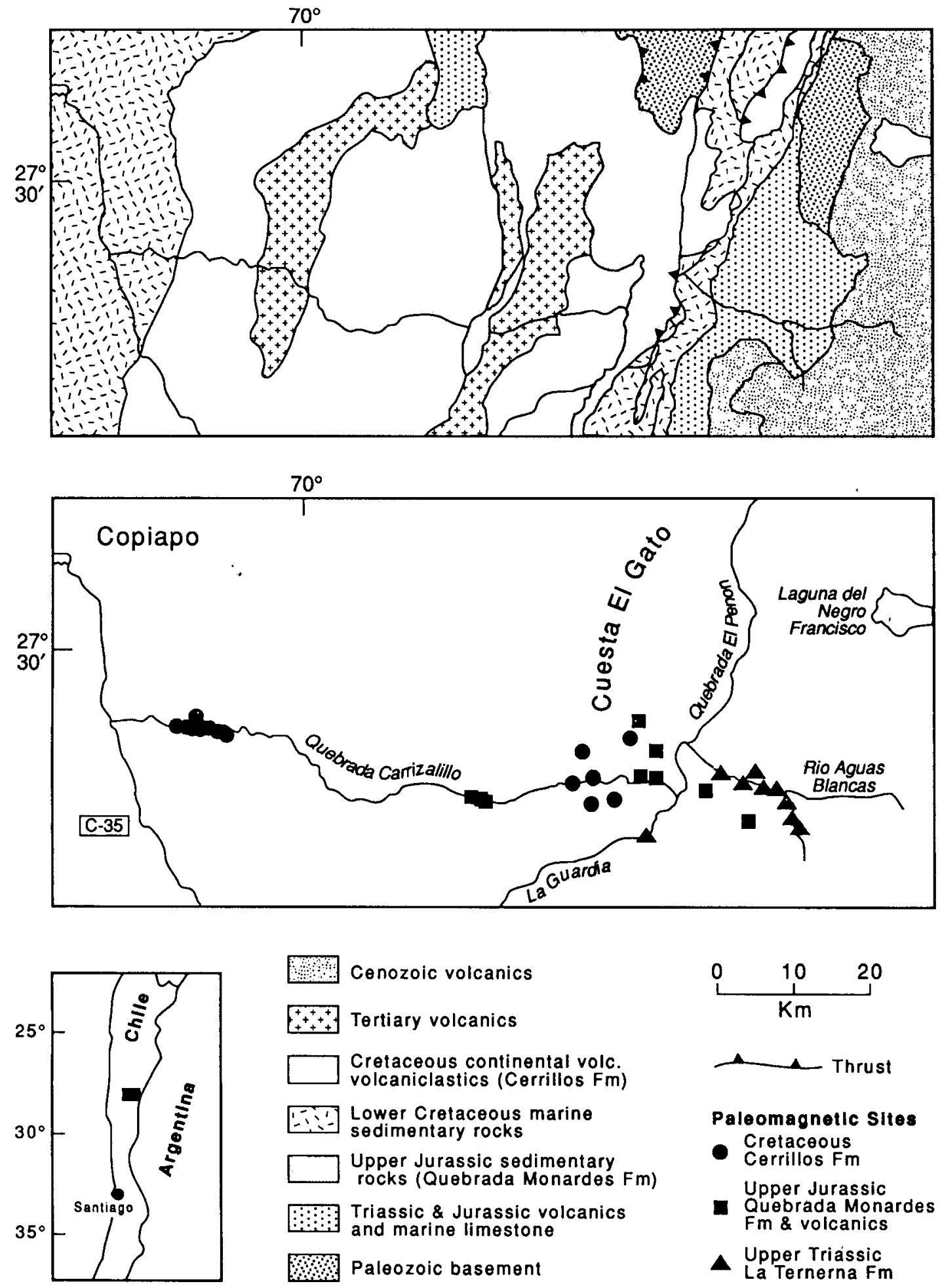

Cenozoic volcanics

Tertiary volcanics

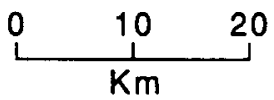

Cretaceous continental volc. volcaniclastics (Cerrillos $\mathrm{Fm}$ )

Lower Cretaceous marine sedimentary rocks

Upper Jurassic sedimentary rocks (Quebrada Monardes Fm)

Triassic \& Jurassic volcanics and marine limestone

Paleozoic basement

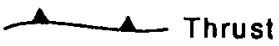

Paleomagnetlc Sites

Cretaceous

Cerrillos Fm

Upper Jurassic

Quebrada Monardes

$\mathrm{Fm}$ \& volcanics

- Upper Triássic

La Ternerna Fm

Fig. 2. Geological sketch map and sample location map.

can craton (Table 5). The Cerrillos Formation was sampled in two distinct localities, one (Cuesta el Gato [CEG]) near the sampling areas of the La Ternera and Quebrada Monardes formations and the other [EDB] about $40 \mathrm{~km}$ to the west. As the magnetic behavior and mean directions of rocks from the two areas are distinct, we report results separately in Table 3 .

La Ternera Formation. Eighteen (of 25) sites displayed very stable remanant magnetiza- tion. Rocks sampled include basaltic and andesitic flows, red lithic volcaniclastic sandstones and breccias, green pyroclastic tuffs, and welded lithic tuffs. Results are divided into three categories: Category $A$ (site 28 ) is characterized by the presence of two opposing magnetic directions. Results of alternating field (AF) and thermal demagnetization suggest that both components reside in magnetite. A component of relatively 
TABLE 1. Paleomagnetic Data for the Upper Triassic La Ternera Formation of Northern Chile

\begin{tabular}{|c|c|c|c|c|c|c|c|c|c|}
\hline \multirow[t]{2}{*}{ site } & \multicolumn{2}{|c|}{ Uncorrected } & \multicolumn{2}{|c|}{ corrected } & \multirow[t]{2}{*}{$\alpha 95$} & \multirow[t]{2}{*}{$\mathbf{R}$} & \multirow[t]{2}{*}{ N/No } & \multicolumn{2}{|c|}{ VGP } \\
\hline & $\mathrm{D}$ & $I$ & $D$ & $\vec{I}$ & & & & Lat & Long \\
\hline $\begin{array}{l}90 \operatorname{tr} 02 \\
90 \operatorname{tr} 03 \\
90 \operatorname{tr} 06 \\
90 \operatorname{tr} 10 \\
90 \operatorname{tr} 13 \\
90 \operatorname{tr} 16 \\
90 \operatorname{tr} 20 \\
90 \operatorname{tr} 22 \\
90 \operatorname{tr} 23 \\
90 \operatorname{tr} 24 \\
90 \operatorname{tr} 25 \\
90 \operatorname{tr} 26 \\
90 \operatorname{tr} 27 \\
90 \operatorname{tr} 28 \\
90 \operatorname{tr} 99 \\
90 \operatorname{tr} 100 \\
90 \operatorname{tr} 101 \\
90 \operatorname{tr} 103 \\
\text { GMU } \\
\text { GMC } \\
\text { MVGP }\end{array}$ & $\begin{array}{r}198.5 \\
35.2 \\
27.6 \\
19.7 \\
232.6 \\
220.6 \\
235.3 \\
32.5 \\
26.1 \\
49.7 \\
219.9 \\
30.4 \\
9.5 \\
208.7 \\
37.0 \\
28.9 \\
49.6 \\
29.0 \\
33.9\end{array}$ & $\begin{array}{l}+41.7 \\
-47.8 \\
-43.4 \\
-41.6 \\
+49.8 \\
+47.9 \\
+56.8 \\
-51.2 \\
-59.5 \\
-49.5 \\
+54.4 \\
-45.5 \\
-48.6 \\
+79.8 \\
-36.7 \\
-43.1 \\
-34.7 \\
-48.5 \\
-49.5\end{array}$ & $\begin{array}{r}187.8 \\
24.6 \\
11.6 \\
19.7 \\
232.6 \\
227.7 \\
246.1 \\
24.5 \\
6.8 \\
39.0 \\
219.9 \\
19.3 \\
9.5 \\
247.2 \\
40.9 \\
31.5 \\
55.5 \\
40.4 \\
33.1\end{array}$ & $\begin{array}{l}+34.9 \\
-46.1 \\
-40.0 \\
-41.6 \\
+49.8 \\
+40.9 \\
+38.8 \\
-58.8 \\
-62.6 \\
-57.2 \\
+54.4 \\
-50.1 \\
-48.6 \\
+66.4 \\
-40.9 \\
-56.0 \\
-45.7 \\
-51.1 \\
-51.1\end{array}$ & $\begin{array}{r}3.9 \\
8.3 \\
11.4 \\
7.7 \\
5.7 \\
4.3 \\
6.6 \\
7.6 \\
5.5 \\
6.9 \\
10.3 \\
9.6 \\
8.7 \\
10.6 \\
5.8 \\
.5 \\
18.4 \\
3.7 \\
5.5 \\
6.5 \\
7.8\end{array}$ & $\begin{array}{l}7.965 \\
6.888 \\
5.859 \\
7.866 \\
7.926 \\
7.959 \\
7.901 \\
7.871 \\
4.979 \\
3.983 \\
5.884 \\
5.899 \\
4.949 \\
4.924 \\
4.977 \\
4.983 \\
3.884 \\
7.968 \\
17.57 \\
17.42 \\
17.15\end{array}$ & $\begin{array}{l}8 / 8 \\
7 / 7 \\
6 / 6 \\
8 / 8 \\
8 / 8 \\
8 / 8 \\
8 / 8 \\
8 / 8 \\
5 / 7 \\
4 / 4 \\
6 / 7 \\
6 / 9 \\
5 / 5 \\
5 / 6 \\
5 / 5 \\
5 / 5 \\
4 / 4 \\
8 / 8 \\
18 \\
18 \\
18\end{array}$ & $\begin{array}{l}78.9 \\
68.2 \\
78.4 \\
71.9 \\
44.4 \\
47.0 \\
30.4 \\
66.5 \\
72.8 \\
55.8 \\
55.4 \\
72.9 \\
81.4 \\
35.1 \\
54.3 \\
62.1 \\
41.2 \\
54.9\end{array}$ & $\begin{array}{l}152.3 \\
205.8 \\
178.1 \\
193.2 \\
217.5 \\
206.1 \\
211.1 \\
237.3 \\
273.8 \\
228.0 \\
222.9 \\
216.1 \\
215.7 \\
242.7 \\
213.9 \\
226.8 \\
213.4 \\
217.1\end{array}$ \\
\hline
\end{tabular}

$D$, and I are site-mean declination and inclination; $\alpha 95$ is radius of circle of $95 \%$ confidence; $R$ is length of vector sum; N/No is number of samples used in calculations/number of samples collected; Lat and Long are south latitude and east longitude of virtual geomagnetic pole (VGP); GMU and GMC are group means uncorrected for tilt and corrected for tilt; MVGP is mean VGP for tilt-corrected directions.

TABLE 2. Paleomagnetic Data for the Lower Jurassic Quebrada Monardes Formation and Associated Volcanic Rocks

\begin{tabular}{|c|c|c|c|c|c|c|c|c|c|}
\hline \multirow[t]{2}{*}{ Site } & \multicolumn{2}{|c|}{ Uncorrected } & \multicolumn{2}{|c|}{ Corrected } & \multirow[t]{2}{*}{$\alpha 95$} & \multirow[t]{2}{*}{$\mathrm{R}$} & \multirow{2}{*}{ N/No } & \multicolumn{2}{|c|}{ VGP } \\
\hline & $\mathrm{D}$ & I & $D$ & I & & & & Iat & Long \\
\hline $\begin{array}{l}90 \mathrm{gm} 14 \\
90 \mathrm{gm} 18 \\
90 \mathrm{gm} 19 \\
90 \mathrm{gm} 47 \\
90 \mathrm{gm} 48 \\
90 \mathrm{gm} 51 \\
90 \mathrm{gm} 54 * \\
90 \mathrm{gm} 55 \\
90 \mathrm{gm} 85 \\
\text { GMU } \\
\text { GMC } \\
\text { MVGP }\end{array}$ & $\begin{array}{r}81.4 \\
18.8 \\
5.6 \\
354.8 \\
20.8 \\
185.8 \\
159.3 \\
222.7 \\
164.2 \\
15.5\end{array}$ & $\begin{array}{l}-38.0 \\
-52.4 \\
-48.6 \\
-36.5 \\
-26.1 \\
+1.3 \\
+59.4 \\
+66.2 \\
+28.9 \\
-40.9\end{array}$ & $\begin{array}{r}35.7 \\
18.1 \\
21.4 \\
19.4 \\
33.5 \\
187.0 \\
197.4 \\
238.8 \\
192.5 \\
23.7\end{array}$ & $\begin{array}{l}-41.5 \\
-29.2 \\
-47.7 \\
-40.8 \\
-19.7 \\
+6.0 \\
+67.2 \\
+60.5 \\
+41.1 \\
-40.9\end{array}$ & $\begin{array}{c}6.4 \\
4.9 \\
14.1 \\
10.36 \\
17.2 \\
6.0 \\
29.6 \\
18.5 \\
20.0 \\
14.1 \\
20.5 \\
12.7\end{array}$ & $\begin{array}{l}5.969 \\
9.903 \\
4.869 \\
4.928 \\
3.898 \\
4.976 \\
2.891 \\
3.883 \\
3.863 \\
7.55 \\
7.04 \\
6.63\end{array}$ & $\begin{array}{l}6 / 6 \\
10 / 10 \\
5 / 7 \\
5 / 5 \\
4 / 4 \\
5 / 6 \\
4 / 6 \\
4 / 4 \\
4 / 4 \\
8 \\
8 \\
8\end{array}$ & $\begin{array}{l}57.7 \\
69.4 \\
71.1 \\
72.0 \\
54.0 \\
64.6 \\
64.0 \\
40.6 \\
78.1\end{array}$ & $\begin{array}{l}202.0 \\
168.6 \\
209.1 \\
191.0 \\
177.9 \\
126.8 \\
264.3 \\
232.9 \\
184.0\end{array}$ \\
\hline
\end{tabular}

\footnotetext{
Column headings are as in Table 1 . VGP is calculated for tilt-corrected directions. * Omitted from final average.
}

TABLE 3. Paleomagnetic Data for the Cretaceous Cerrillos Formation

\begin{tabular}{|c|c|c|c|c|c|c|c|c|c|}
\hline \multirow[t]{2}{*}{ site } & \multicolumn{2}{|c|}{ Uncorrected } & \multicolumn{2}{|c|}{ Corrected } & \multirow[t]{2}{*}{$\alpha 95$} & \multirow[t]{2}{*}{$\mathbf{R}$} & \multirow[t]{2}{*}{ N/No } & \multicolumn{2}{|c|}{ VGP } \\
\hline & $\mathrm{D}$ & $\mathrm{I}$ & $\mathrm{D}$ & $\bar{I}$ & & & & Lat & Long \\
\hline $\begin{array}{l}90 \operatorname{cs} 33 \\
90 \operatorname{cs} 34 \\
90 \operatorname{cs} 41 \\
90 \operatorname{cs} 42 \\
90 \operatorname{cs} 44 \\
90 \operatorname{cs} 45 \\
\text { GMU } \\
\text { GMC } \\
\text { MVGP }\end{array}$ & $\begin{array}{l}214.6 \\
192.2 \\
210.6 \\
203.7 \\
162.1 \\
184.1 \\
191.7\end{array}$ & $\begin{array}{r}+54.2 \\
+41.5 \\
+54.1 \\
+25.8 \\
+63.2 \\
+64.6 \\
51.8\end{array}$ & $\begin{array}{l}161.0 \\
202.3 \\
206.8 \\
202.5 \\
208.3 \\
208.8 \\
201.6\end{array}$ & $\begin{array}{l}+71.2 \\
+48.1 \\
+24.3 \\
+11.2 \\
+72.4 \\
+57.8 \\
+48.5\end{array}$ & $\begin{array}{r}10.2 \\
6.6 \\
7.7 \\
8.1 \\
4.9 \\
5.5 \\
15.4 \\
22.9 \\
16.2\end{array}$ & $\begin{array}{c}5.886 \\
4.970 \\
6.904 \\
4.955 \\
14.77 \\
3.989 \\
5.75 \\
5.47 \\
5.70\end{array}$ & $\begin{array}{l}6 / 6 \\
5 / 7 \\
7 / 10 \\
5 / 7 \\
15 / 16 \\
4 / 4 \\
6 \\
6 \\
6\end{array}$ & $\begin{array}{l}59.7 \\
78.4 \\
63.0 \\
63.8 \\
67.8 \\
70.9\end{array}$ & $\begin{array}{l}222.6 \\
184.0 \\
223.0 \\
172.7 \\
325.6 \\
281.7\end{array}$ \\
\hline \multicolumn{10}{|c|}{ Elisa De Bordo } \\
\hline $\begin{array}{l}90 \text { eb65 } \\
90 \text { eb6 } 6\end{array}$ & $\begin{array}{l}41.6 \\
38.4\end{array}$ & $\begin{array}{l}-52.4 \\
-48.1\end{array}$ & $\begin{array}{l}29.8 \\
35.5\end{array}$ & $\begin{array}{l}-58.3 \\
-57.9\end{array}$ & $\begin{array}{l}8.7 \\
7.1\end{array}$ & $\begin{array}{l}5.917 \\
6.917\end{array}$ & $\begin{array}{l}6 / 6 \\
7 / 7\end{array}$ & $\begin{array}{l}53.9 \\
56.3\end{array}$ & $\begin{array}{l}218.9 \\
211.8\end{array}$ \\
\hline
\end{tabular}


TABLE 3. (continued)

Elisa De Bordo

\begin{tabular}{|c|c|c|c|c|c|c|c|c|c|}
\hline \multirow[t]{2}{*}{ Site } & \multicolumn{2}{|c|}{ Uncorrected } & \multicolumn{2}{|c|}{ Corrected } & \multirow[t]{2}{*}{$\alpha 95$} & \multirow[t]{2}{*}{$\mathrm{R}$} & \multirow[t]{2}{*}{$\mathrm{N} / \mathrm{No}$} & \multicolumn{2}{|c|}{ VGP } \\
\hline & $\bar{D}$ & $I$ & $\mathrm{D}$ & $\vec{I}$ & & & & Lat & Long \\
\hline $\begin{array}{l}90 \mathrm{eb} 69 \\
90 \mathrm{eb} 70 \\
90 \mathrm{eb} 71 \\
90 \mathrm{eb} 72 \\
90 \mathrm{eb} 73 \\
90 \mathrm{eb} 74 \\
\text { GMU } \\
\text { GMC } \\
\text { MVGP }\end{array}$ & $\begin{array}{l}43.4 \\
71.0 \\
48.2 \\
59.2 \\
50.7 \\
43.8 \\
46.8\end{array}$ & $\begin{array}{l}-46.3 \\
-24.0 \\
-44.3 \\
-43.2 \\
-36.9 \\
-42.2 \\
-45.0\end{array}$ & $\begin{array}{r}358.0 \\
46.2 \\
351.4 \\
67.7 \\
51.2 \\
46.0 \\
26.1\end{array}$ & $\begin{array}{l}-46.9 \\
-50.2 \\
-44.1 \\
-67.6 \\
-61.9 \\
-62.2 \\
-59.7\end{array}$ & $\begin{array}{r}5.9 \\
27.4 \\
15.4 \\
9.9 \\
14.3 \\
10.7 \\
5.2 \\
13.0 \\
5.8\end{array}$ & $\begin{array}{l}7.921 \\
3.754 \\
4.843 \\
5.893 \\
3.929 \\
3.959 \\
6.96 \\
6.73 \\
6.94\end{array}$ & $\begin{array}{l}8 / 8 \\
4 / 5 \\
5 / 6 \\
6 / 6 \\
4 / 5 \\
4 / 7 \\
7 \\
7 \\
7\end{array}$ & $\begin{array}{l}51.7 \\
22.5 \\
47.2 \\
37.4 \\
43.5 \\
50.7\end{array}$ & $\begin{array}{l}210.3 \\
202.6 \\
209.2 \\
211.6 \\
202.7 \\
205.4\end{array}$ \\
\hline
\end{tabular}

Column headings are as in Table 1. VGP is calculated for uncorrected directions.

TABLE 4. Additional Data for Mesozoic Formations in North Central Chile

\begin{tabular}{|c|c|c|c|c|c|}
\hline site & Lat & Long & Attitude & Demag Range & Lithology \\
\hline \multicolumn{6}{|c|}{ Upper Triassic La Ternera Formation } \\
\hline $90 \operatorname{tro} 2$ & $27^{\circ} 42 \cdot 33^{\prime \prime}$ & $69^{\circ} 21^{\prime} 54^{\prime \prime}$ & $\mathrm{N} 40^{\circ} \mathrm{E} / 15^{\circ} \mathrm{SE}$ & $410-590$ & welded tuff \\
\hline $90 \operatorname{tr} 03$ & $27^{\circ} 41^{\prime} 39^{\prime \prime}$ & $69^{\circ} 22^{\prime} 12^{\prime \prime}$ & $\mathrm{N} 40^{\circ} \mathrm{E} / 10^{\circ} \mathrm{SE}$ & $26-100$ & hornblende andesite \\
\hline $90 \operatorname{tr} 06$ & $27^{\circ} 40^{\prime} 36^{\prime \prime}$ & $69^{\circ} 22^{\prime} 42 "$ & $\mathrm{~N} 30^{\circ} \mathrm{E} / 18^{\circ} \mathrm{SE}$ & $18-100$ & andesite breccia \\
\hline $90 \operatorname{tr} 13$ & $27^{\circ} 39^{\prime} 51 "$ & $69^{\circ} 24^{\prime} 15^{\prime \prime}$ & ーーーーーーーーーー & $40-100$ & red volcanic breccia \\
\hline $90 \operatorname{tr} 16$ & $27^{\circ} 38^{\prime} 42^{\prime \prime}$ & $69^{\circ} 28^{\prime} 03^{\prime \prime}$ & $\mathrm{NO} 0^{\circ} \mathrm{E} / 10^{\circ} \mathrm{W}$ & $25-100$ & welded red tuff \\
\hline $90 \operatorname{tr} 20$ & $27^{\circ} 38^{\prime} 42^{\prime \prime}$ & $69^{\circ} 28^{\prime} 03^{\prime \prime}$ & $\mathrm{NO} 0^{\circ} \mathrm{E} / 20^{\circ} \mathrm{W}$ & $30-100$ & fine grained basalt \\
\hline $90 \operatorname{tr} 22$ & $27^{\circ} 40^{\prime} 00^{\prime \prime}$ & $69^{\circ} 23^{\prime} 40^{\prime \prime}$ & $\mathrm{N} 50^{\circ} \mathrm{W} / 10^{\circ} \mathrm{E}$ & $30-100$ & fine grained basalt \\
\hline $90 \operatorname{tr} 23$ & $27^{\circ} 40^{\prime} 00^{\prime \prime}$ & $69^{\circ} 23 \cdot 41^{\prime \prime}$ & $\mathrm{N} O 0^{\circ} \mathrm{E} / 11^{\circ} \mathrm{E}$ & $420-590$ & fine grained basalt \\
\hline $90 t r 24$ & $27^{\circ} 40^{\prime} 00^{\prime \prime}$ & $69^{\circ} 23^{\prime} 00^{\prime \prime}$ & $69^{\circ} 23^{\prime} 41^{\prime \prime}$ & $30-60$ & fine grained andesite \\
\hline $90 \operatorname{tr} 25$ & $27^{\circ} 40^{\prime} 00^{\prime \prime}$ & $69^{\circ} 23^{\prime} 41^{\prime \prime}$ & $--m-----$ & $30-100$ & red welded tuff \\
\hline $90 \operatorname{tr26}$ & $27^{\circ} 40^{\prime} 00^{\prime \prime}$ & $69^{\circ} 23^{\prime} 41^{\prime \prime}$ & $\mathrm{N} 00^{\circ} \mathrm{E} / 11^{\circ} \mathrm{E}$ & $20-50$ & green tuff \\
\hline $90 \operatorname{tr} 28$ & $27^{\circ} 39 \cdot 30^{\prime \prime}$ & $69^{\circ} 26^{\prime} 00^{\prime \prime}$ & $\mathrm{NO} 0^{\circ} \mathrm{E} / 17^{\circ} \mathrm{W}$ & $26-85$ & fine grained basalt \\
\hline $90 \operatorname{tr99}$ & $27^{\circ} 43 \cdot 45^{\prime \prime}$ & $69^{\circ} 37^{\prime} 15^{\prime \prime}$ & $N 70^{\circ} \mathrm{W} / 13^{\circ} \mathrm{N}$ & $340-660$ & red porphyritic basalt \\
\hline $90 \operatorname{tr} 100$ & $27^{\circ} 43^{\prime} 45^{\prime \prime}$ & $69^{\circ} 37^{\prime} 15^{\prime \prime}$ & $\mathrm{N} 70^{\circ} \mathrm{W} / 13^{\circ} \mathrm{N}$ & $410-660$ & red porphyritic basalt \\
\hline $90 t r 101$ & $27^{\circ} 43^{\prime} 45^{\prime \prime}$ & $69^{\circ} 37 \cdot 15^{\prime \prime}$ & $\mathrm{N} 70^{\circ} \mathrm{W} / 13^{\circ} \mathrm{N}$ & $3-65$ & fine grained basalt \\
\hline $90 \operatorname{tr} 103$ & $27^{\circ} 43^{\prime} 45^{\prime \prime}$ & $69^{\circ} 37^{\prime} 15^{\prime \prime}$ & $\mathrm{N} 70^{\circ} \mathrm{W} / 13^{\circ} \mathrm{N}$ & $30-100$ & fine grained basalt \\
\hline
\end{tabular}

Lower Jurassic Quebrada Monardes Formation

\begin{tabular}{|c|c|c|c|c|c|}
\hline $90 \mathrm{gm} 14$ & $27^{\circ} 39 \cdot 24 "$ & $69^{\circ} 26^{\prime} 50^{\prime \prime}$ & $\mathrm{N} 55^{\circ} \mathrm{E} / 50^{\circ} \mathrm{SE}$ & $420-630$ & red sandstone \\
\hline 90 qm18 & $27^{\circ} 39^{\prime} 12^{\prime \prime}$ & $69^{\circ} 26^{\prime} 30^{\prime \prime}$ & $\mathrm{N} 70^{\circ} \mathrm{W} / 23^{\circ} \mathrm{S}$ & $350-580$ & red sandstone \\
\hline 90 qm19 & $27^{\circ} 38^{\prime} 09^{\prime \prime}$ & $69^{\circ} 28^{\prime} 39^{\prime \prime}$ & $\mathrm{N} 10^{\circ} \mathrm{E} / 14^{\circ} \mathrm{N}$ & $350-610$ & red sandstone \\
\hline $90 \mathrm{qm} 47$ & $27^{\circ} 38 \cdot 30^{\prime \prime}$ & $69^{\circ} 35 \cdot 30^{\prime \prime}$ & $\mathrm{N} 15^{\circ} \mathrm{E} / 30^{\circ} \mathrm{N}$ & $20-100$ & red sandstone \\
\hline 90 qm48 & $27^{\circ} 38^{\prime} 30^{\prime \prime}$ & $69^{\circ} 35^{\prime} 30^{\prime \prime}$ & $\mathrm{N} 15^{\circ} \mathrm{E} / 30^{\circ} \mathrm{N}$ & $20-90$ & $\begin{array}{c}\text { volcaniclastic } \\
\text { sandstone }\end{array}$ \\
\hline 90 qm51 & $27^{\circ} 38^{\prime} 30^{\prime \prime}$ & $69^{\circ} 35^{\prime} 30^{\prime \prime}$ & $\mathrm{N} 20^{\circ} \mathrm{E} / 20^{\circ} \mathrm{N}$ & $\begin{array}{r}40-100 \\
530-630 *\end{array}$ & hornblende andesite \\
\hline $90 \operatorname{qm} 54$ & $27^{\circ} 38^{\prime} 28^{\prime \prime}$ & $69^{\circ} 35^{\prime} 29^{\prime \prime}$ & $\mathrm{N} 20^{\circ} \mathrm{E} / 20^{\circ} \mathrm{N}$ & $20-70$ & $\begin{array}{c}\text { volcaniclastic } \\
\text { sandstone }\end{array}$ \\
\hline 90 qm5 5 & $27^{\circ} 38^{\prime} 28^{\prime \prime}$ & $69^{\circ} 35^{\prime} 29^{\prime \prime}$ & $\mathrm{N} 05^{\circ} \mathrm{E} / 10^{\circ} \mathrm{N}$ & $\begin{array}{r}20-100 \\
630-675 *\end{array}$ & red sandstone \\
\hline $90 \mathrm{qm} 85$ & $27^{\circ} 30^{\prime} 39^{\prime \prime}$ & $69^{\circ} 34^{\prime} 40^{\prime \prime}$ & $\mathrm{N} 15^{\circ} \mathrm{E} / 40^{\circ} \mathrm{N}$ & $550-650 *$ & red sandstone \\
\hline
\end{tabular}

Cretaceous Cerrillos Formation: Cordon El Gato

\begin{tabular}{|c|c|c|c|c|c|}
\hline $90 \operatorname{cs} 33$ & $27^{\circ} 39^{\prime} 25^{\prime \prime}$ & $69^{\circ} 39^{\prime} 45^{\prime \prime}$ & $\mathrm{N} 12^{\circ} \mathrm{W} / 30^{\circ} \mathrm{N}$ & $\begin{array}{c}500-580 \\
40-85\end{array}$ & fine grained andesite \\
\hline $90 \operatorname{cs} 34$ & $27^{\circ} 39 \cdot 10^{\prime \prime}$ & $69^{\circ} 39^{\prime} 20^{\prime \prime}$ & $\mathrm{N} 50^{\circ} \mathrm{E} / 12^{\circ} \mathrm{N}$ & $20-90$ & fine grained \\
\hline
\end{tabular}


TABLE 4. (continued)

Cretaceous Cerrillos Formation: Cordon El Gato

\begin{tabular}{|c|c|c|c|c|c|}
\hline $900 s 41$ & $27^{\circ} 38^{\prime} 35^{\prime \prime}$ & $69^{\circ} 37^{\prime} 43^{\prime \prime}$ & $\mathrm{N} 70^{\circ} \mathrm{W} / 30^{\circ} \mathrm{s}$ & $540-610^{*}$ & porphyritic andesite \\
\hline $90 \operatorname{cs} 42$ & $27^{\circ} 37^{\prime} 43^{\prime \prime}$ & $69^{\circ} 37^{\prime} 43^{\prime \prime}$ & $\mathrm{N} 85^{\circ} \mathrm{W} / 15^{\circ} \mathrm{S}$ & $40-100$ & porphyritic andesite \\
\hline $90 \operatorname{cs} 44$ & $27^{\circ} 39 \cdot 30^{\prime \prime}$ & $69^{\circ} 36^{\prime} 30^{\prime \prime}$ & $\mathrm{N} 30^{\circ} \mathrm{E} / 20^{\circ} \mathrm{N}$ & $\begin{array}{c}550-640, * \\
40-90\end{array}$ & prophyritic andesite \\
\hline $90 \operatorname{cs} 45$ & $27^{\circ} 39^{\prime} 30^{\prime \prime}$ & $69^{\circ} 36^{\prime} 30^{\prime \prime}$ & $\mathrm{N} 10^{\circ} \mathrm{W} / 15^{\circ} \mathrm{S}$ & $\begin{array}{c}500-580 \\
20-100\end{array}$ & fine grained andesite \\
\hline
\end{tabular}

Upper Cretaceous Cerrillos Formation: Eliza de Bordo

\begin{tabular}{|c|c|c|c|c|c|}
\hline $90 e b 65$ & $27^{\circ} 34 \cdot 40^{\prime \prime}$ & $70^{\circ} 07 \cdot 00^{\prime \prime}$ & $\mathrm{NO} 0^{\circ} \mathrm{E} / 10^{\circ} \mathrm{E}$ & $40-100$ & andesite flow \\
\hline $90 e b 66$ & $27^{\circ} 34^{\prime} 40^{\prime \prime}$ & $70^{\circ} 08^{\prime} 00^{\prime \prime}$ & $\mathrm{N} 45^{\circ} \mathrm{W} / 12^{\circ} \mathrm{NE}$ & $\begin{array}{l}350-580, * \\
25-65\end{array}$ & mafic flow \\
\hline $90 e b 60$ & $27^{\circ} 34^{\prime} 30^{\prime \prime}$ & $70^{\circ} 12^{\prime} 00^{\prime \prime}$ & $\mathrm{N} 20^{\circ} \mathrm{E} / 40^{\circ} \mathrm{SE}$ & $\begin{array}{c}340-580, * \\
30-100\end{array}$ & andesite \\
\hline $90 e b 70$ & $27^{\circ} 33^{\prime} 10^{\prime \prime}$ & $70^{\circ} 11^{\prime} 55^{\prime \prime}$ & $\mathrm{N} 20^{\circ} \mathrm{E} / 40^{\circ} \mathrm{SE}$ & $20-75$ & volcanoclastic \\
\hline 90 eb 71 & $27^{\circ} 34^{\prime} 20^{\prime \prime}$ & $70^{\circ} 12 \cdot 30^{\prime \prime}$ & $\mathrm{N} 20^{\circ} \mathrm{E} / 52^{\circ} \mathrm{SE}$ & $20-80$ & Iimestone \\
\hline $90 \mathrm{eb} 72$ & $27^{\circ} 34^{\prime} 40^{\prime \prime}$ & $70^{\circ} 11 \cdot 30^{\prime \prime}$ & $\mathrm{N} 40^{\circ} \mathrm{W} / 25^{\circ} \mathrm{NE}$ & $\begin{array}{c}30-90 \\
400-500 *\end{array}$ & prophyritic andesite \\
\hline $90 \mathrm{eb} 73$ & $27^{\circ} 34 \cdot 35^{\prime \prime}$ & $70^{\circ} 11 \cdot 50^{\prime \prime}$ & $\mathrm{N} 40^{\circ} \mathrm{W} / 25^{\circ} \mathrm{NE}$ & $350-570 *$ & andesite breccia \\
\hline 90 eb 74 & $27^{\circ} 34^{\prime} 15^{\prime \prime}$ & $70^{\circ} 09^{\prime} 25^{\prime \prime}$ & $\mathrm{N} 50^{\circ} \mathrm{W} / 20^{\circ} \mathrm{NE}$ & $340-560 *$ & porphyritic andesite \\
\hline
\end{tabular}

Lat and Long are south latitude and west longitude of site. Attitude is given in standard geological notation (strike/dip). Demag range indicates range of demagnetization steps used in line fitting to determined sample directions. Thermal demagnetization is shown by asterisk; other numbers are alternating field demagnetization range, in milliteslas. Note that some sites contain samples whose directions were determined by both alternating field demagnetization and thermal demagnetization or by a combination of the two (see text).

low stability, directed $\mathrm{NE}$ and up, is removed by $20 \mathrm{mT}$ and $410^{\circ} \mathrm{C}$. A more stable SW and down component then is removed between 20 and $80 \mathrm{mT}$ and between $410^{\circ}$ and $580^{\circ} \mathrm{C}$. Table 1 gives the directions of the higher-stability component; the less stable component probably represents a VRM acquired in the present geomagnetic field. Category B sites $(2,3,6,10,13,16,20$, 22 , and $24-27$ ) have a single, well-defined component of magnetization residing mostly in magnetite but also present in hematite. Both polarities are present. The direction of the hematite component (isolated between $580^{\circ}$ and $650^{\circ} \mathrm{C}$ ) is indistinguishable at $95 \%$ confidence from the component isolated by AF (presumably carried by magnetite). AF results are reported in Table 1.

Category C (sites 99 and 100) has normal polarity residing in hematite. Site 99 displays a single component removed between $350^{\circ}$ and $640^{\circ} \mathrm{C}$. Site 100 showed a linear thermal demagnetization trend which diverged from the origin, suggesting that a small component of magnetization may remain after demagnetization to $670^{\circ} \mathrm{C}$. Seven sites from the la Ternera Formation are not included in Table 1 . Four of these are coarse grained sandstones or obviously altered andesites that displayed erratic demagnetization paths. Three other sites, in unaltered volcan- ics, have stable single-component magnetizations but high within site scatter, possibly indicating lightning strikes, postmagnetization rheomorphic flow, orientation errors, or other unknown effects.

Quebrada Monardes red beds. Site mean directions for the nine reasonably well behaved sites that we have completed from this unit are listed in Table 2. These directions were obtained from red fluvial and aeolian sandstones and from one andesitic flow. Results are divided into four categories:

Category A (sites 14, 18, and 19) have a single-component normal polarity magnetization carried by hematite, isolated from $350^{\circ}$ to $650^{\circ} \mathrm{C}$.

Category B (site 85) has a NW and up component removed by $425^{\circ} \mathrm{C}$ and a stable SW and down component isolated between $425^{\circ}$ and $670^{\circ} \mathrm{C}$. Alternating field demagnetization up to loom had little effect on this site, suggesting that both components reside in hematite. Table 2 includes only results from the component with high unblocking temperature.

category C (sites 47 and 48 ) is characterized by $\mathrm{a} \mathrm{NE}$ and up component of magnetization carried by magnetite. Samples responded well to $A F$ demagnetization.

Category D (sites 51, 54, and 55) has a NE 

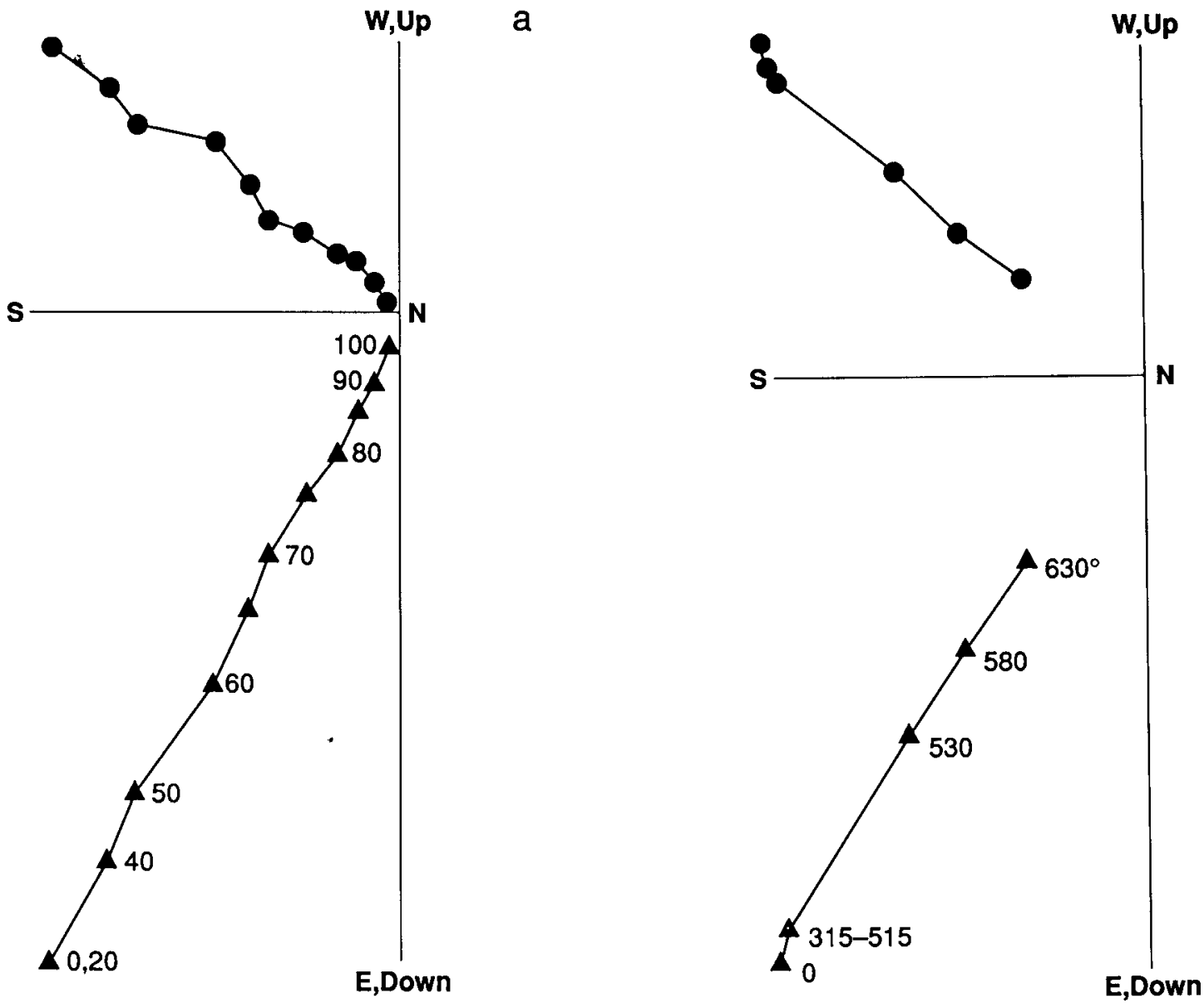

Fig. 3. Orthogonal diagrams illustrating response to laboratory demagnetization. (a) Alternating field (AF) demagnetization of reverse-polarity red welded tuff from La Ternera Formation. Peak demagnetizing field (in milliteslas) indicated. Dots represent map view; triangles represent vertical section. (b) Thermal (Th) demagnetization of same rock unit. Temperatures (in degrees Celsius) are indicated. (c) Th demagnetization of normal-polarity red sandstone from Quebrada Monardes Formation. (d) Th and AF demagnetization of andesite from Cerrillos Formation, Cuesta el Gato locality. Sample was first demagnetized to $80 \mathrm{mT}$, then from $500^{\circ}$ to $630^{\circ} \mathrm{C}$. (e) $\mathrm{AF}$ demagnetization of mafic flow from Cerrillos Formations, Eliza de Bordo locality.

and up component of magnetization, removed by $60 \mathrm{mT}$ and $340^{\circ} \mathrm{C}$, and $\mathrm{a} S W$ and down component isolated between 60 and $100 \mathrm{mT}$ or between 20 and 70 $\mathrm{mT}$. Site $90 \mathrm{qm} 54$ has a circle of $95 \%$ confidence that exceeds $20^{\circ}$ and has not been used in group mean calculations.

The remaining Quebrada Monardes sites studied to date display very complicated results. For instance, four sites located stratigraphically below site $90 \mathrm{gm} 54$ were resistant (failed to change direction) to $\mathrm{AF}$ (to $100 \mathrm{mT}$ ) and thermal (to $680^{\circ} \mathrm{C}$ ) demagnetization. Two other sites, located near site 85 in thinly bedded mudstone and limestone, had linear demagnetization paths but highly discordant directions. Work is still in progress on our remaining Quebrada Monardes collection which, owing to the enthusiasm of the second author during the first field season, is still quite substantial.

Cerrillos Formation. Results from the Cerrillos Formation are discussed by locality. The western locality, Elisa de Bordo (EDB), consists of volcanic and sedimentary rocks that dip $30^{\circ}-$ $45^{\circ}$ to the $\mathrm{NE}$ and $\mathrm{SW}$. The eastern locality, Cuesta el Gato (CEG), located in the same gen- eral area as the La Ternera and Quebrada Monardes sites, includes andesite flows, breccias, and volcaniclastic rocks.

EDB. Site mean directions for eight (of 10) sites and the expected direction are given in Table 3. All sites have normal polarity. Results are divided into two categories. Category A (sites $65,66,68$ and 69) has a single component of magnetization, carried mostly by magnetite but also present in hematite. Pilot samples demagnetized thermally had fairly uniform unblocking temperatures of $550^{\circ}-$ $620^{\circ} \mathrm{C}$; AF pilots were magnetically cleaned (reached stable end-points) between 20 and 100 $\mathrm{mT}$.

Category $B$ (sites $70,71,73$, and 74 ) is similar to category $A$ but with the addition of one or both of the following: (1) a streaked distribution of sample directions and (2) multiple components of magnetization. Site $90 \mathrm{eb} 70$ had a large circle of confidence and highly divergent mean direction (Table 1); hence it was not used to calculate the group mean.

CEG. Site mean directions for the six stable sites collected at this locality are 

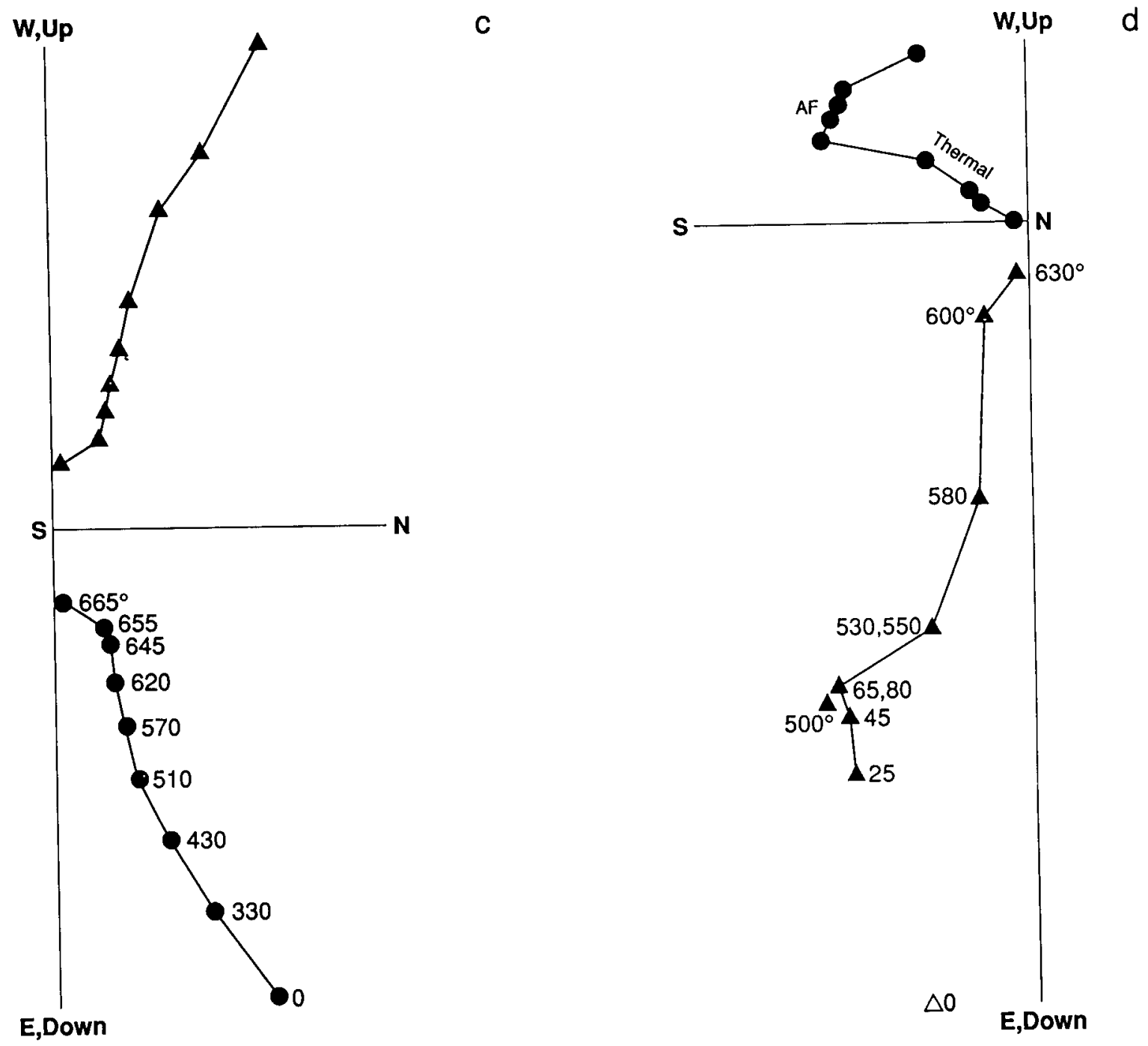

Fig. 3. (continued)

given in Table 3; several other sites yielded unstable or ambiguous magnetizations. Many problematic sites in this locality are situated near an Eocene granodiorite and probably have been affected by local thermal or hydrothermal events of unknown magnitude or duration. The complicated magnetic behavior of these rocks makes their directions suspect. All samples are reversely magnetized. Acceptable results are divided into two categories.

Category A (sites $33,42,44$, and 45) has linear $\mathrm{AF}$ and thermal demagnetization trajectories, with the remanent magnetization (RM) carried by magnetite. Most magnetizations were demagnetized by $100 \mathrm{mT}$ and $580^{\circ} \mathrm{C}$, although persistence of some remanence above these levels shows that hematite is present.

Category B (sites 34 and 41 ) contains sites with multiple components of magnetization residing in both magnetite and hematite. AF (up to $100 \mathrm{mT}$ ) followed by thermal $580^{\circ}$ to $640^{\circ} \mathrm{C}$ ) demagnetization isolated different components of magnetization in some specimens. Generally, thermal demagnetization between $500^{\circ}$ and $630^{\circ} \mathrm{C}$ isolated a component which trended toward the origin. For other samples, AF demagnetization (to $100 \mathrm{mT}$ ) identified a similar trend. The results given in Table 3 were obtained from selected, relatively uncomplicated demagnetiza- tion trajectories that trended toward the origin.

Field stability tests. Field stability tests were used to help determine whether components of magnetization were primary or secondary. Tests used in this study include the fold test, consistency of reversal, and a conglomerate test.

Tilt corrections for the la Ternera Formation are too small to allow a meaningful fold test. There is a slight increase in scatter upon tilt correction seen in Table 1 and Figures $4 \mathrm{a}$ and $4 \mathrm{~b}$, although the increase is not significant at 958 confidence according to Cox [1969]. The increase probably is the result of our inability to measure the dip with high accuracy and of incorrectly applying tilt corrections for what was actually an original dip. Mean normal $(N)$ and reverse (R) directions are antipodal at 95\% confidence. RM of specimens from the $N$ and $R$ subgroups displayed similar stability during laboratory demagnetization. No correlation was found between polarity and lithology, color, location, or magnetic mineralogy. As there is no geological reason to suspect remagnetization we use the tilt-corrected mean direction for further analysis.

The Quebrada Monardes Formation also has $\mathrm{N}$ and $R$ sites with nearly antipodal mean direc- 


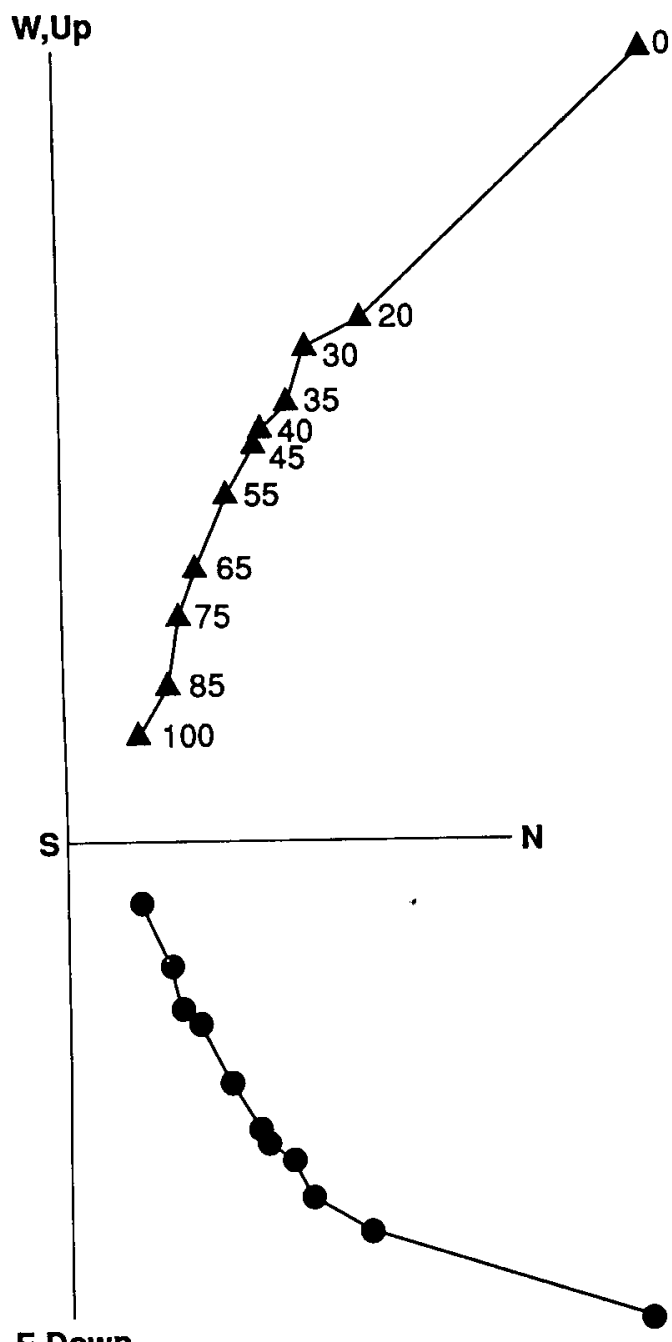

E,Down

Fig. 3. (continued)

tions, plus positive conglomerate and fold tests. For the conglomerate test, clasts were collected from three conglomerate beds (near sites 47, 51, and 54). Most are volcanic andesites and probably are Triassic and/or Jurassic in age. Magnetization was stable during $A F$ and thermal demagnetization; the directions recovered were random. As with the La Ternera Formation, a formal fold test for the Quebrada Monardes Formation is infeasible. However, the increase in precision parameter that results when the tilt correction is made $(7.3$ to 14.1) is significant at 958 confidence, according to $\operatorname{Cox}[1969]$. The weight of evidence thus supports a primary origin for the Quebrada Monardes remanence. Note that in both the La Terera and Quebrada Monardes sections, dips are so gentle that mean directions with and without tilt correction are indistinguishable at $95 \%$ confidence.

In contrast, both areas of Cerrillos rocks appear to be remagnetized. Directions from the CEG locality become more dispersed and less circular when the tilt correction is applied (Figures $6 \mathrm{a}$ and $6 \mathrm{~b}$ and Table 3 ). Rocks at the EDB locality appear completely unaltered in the field, but Figures $7 \mathrm{a}$ and $7 \mathrm{~b}$ and Table 3 strongly indicate that they also have been remagnetized after tilting. No field stability tests were possible at either Cerrillos locality.
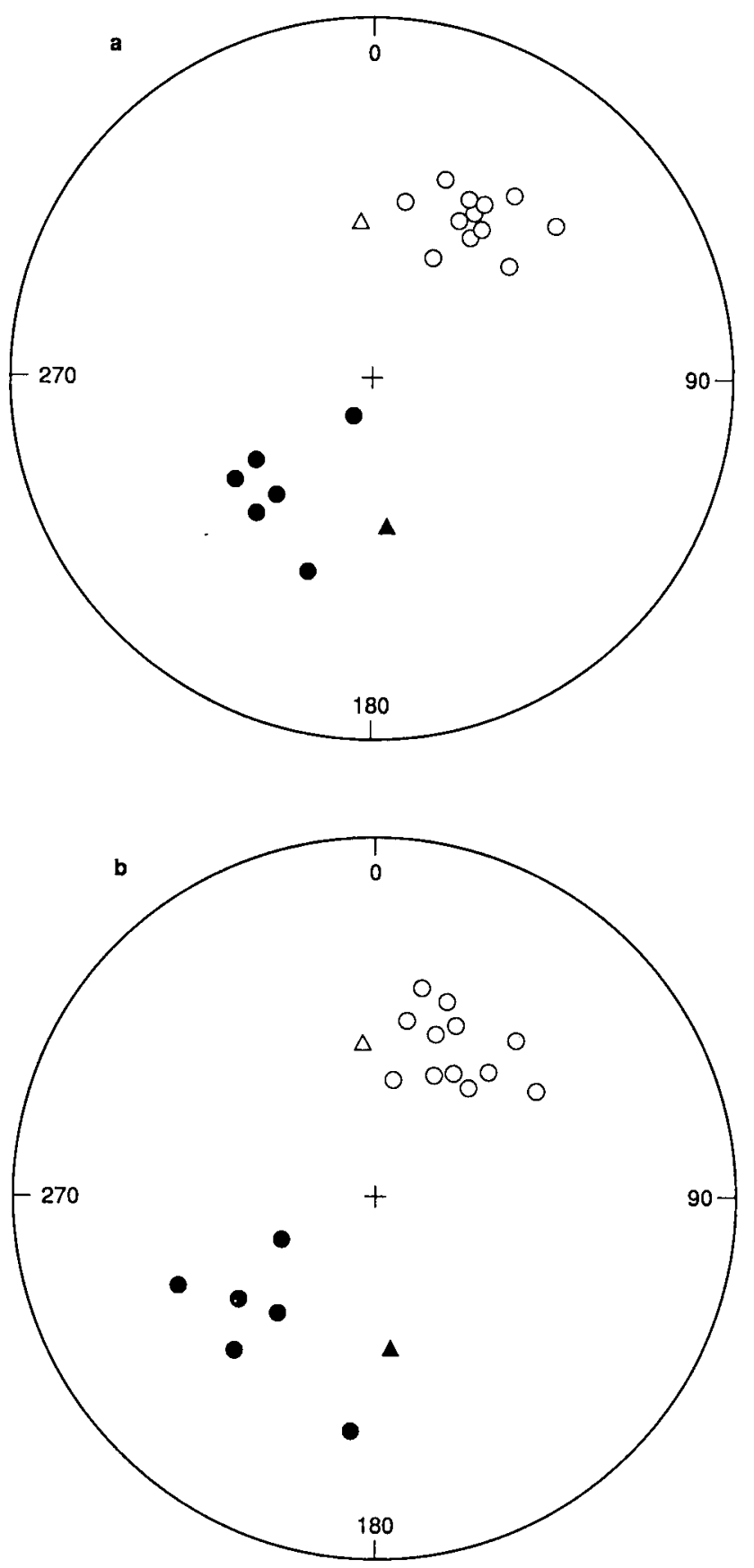

Fig. 4. Equal-area plot of site mean directions of remanent magnetization from La Ternera Formation: (a) in situ and (b) corrected for tilt. Solid/open symbols indicate lower/upper hemisphere. Triangles show expected direction.

\section{South American Reference Poles}

Since our ultimate objective is to determine whether or not our sampling area has moved with respect to stable South America, we must next compare the mean poles of Tables 1-3 with appropriate reference poles for the South American craton. Table 5 summarizes the reference poles used in this study. For the Triassic we calculated the mean of five separate formation poles (listed in Table 5), and for the Jurassic we used results of a similar combination, from Beck [1988]. Although the Cerrillos is assigned a Cretaceous age, its magnetization is clearly 

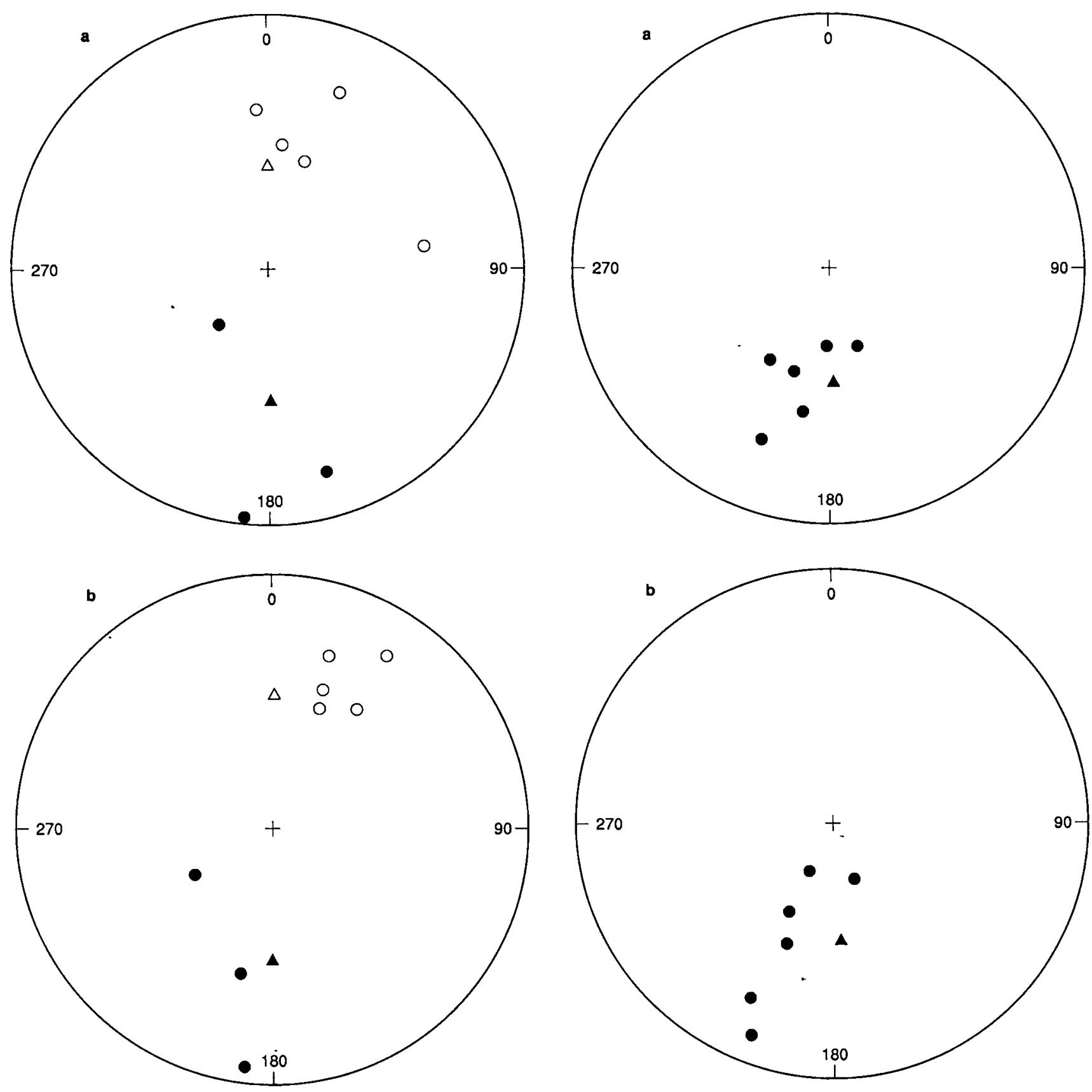

Fig. 5. Quebrada Monardes Formation. See caption for Figure 4 .

younger; presumably early Tertiary. Unfortunately, there are no reliable early Tertiary reference poles for stable South America (see compilation by Irving and Irving, 1982). For that reason we used the early Tertiary (44-54 Ma) North American (NA) pole of Diehl et al. [1983], rotated into South America (SA) coordinates using the reconstruction parameters of Engebretson et al. [1985]. To perform the rotation, we used the $37 \mathrm{Ma}$ SA/NA stage pole, then prorated the rotation on the $66 \mathrm{Ma}$ stage pole to $44 \mathrm{Ma}$. We have arbitrarily assigned an "error circle" of $10^{\circ}$ to this rotated pole. The nominal error circle on the Diehl et al. (1983) pole is only $3.0^{\circ}$, but this clearly understates the probable error on our ultimate objective, that is, to establish a "reference pole" for a poorly

Fig. 6. Cerrillos Formation, Cuesta el Gato locality. See caption for Figure 4.

specified time in the early Tertiary for South America, by using a reference pole from another continent.

Note that none of the reference poles listed in Table 5 is significantly different from the present spin axis at $95 \%$ conifidence, and in fact the maximum angular difference between the Triassic and Jurassic poies of Table 5, commonly used South American Cretaceous reference poles [Bellieni et al., 1983; Butler et al., 1991], and the rotated rertiary pole of Diehl et al. [1983] is only $13.4^{\circ}$. It seems clear that apparent polar wander (APW) relative to South America during the past $230 \mathrm{~m} . \mathrm{y}$. has been remarkably slight and thus that any motion of South America relative to the spin axis has been almost completely along lines of latitude. 


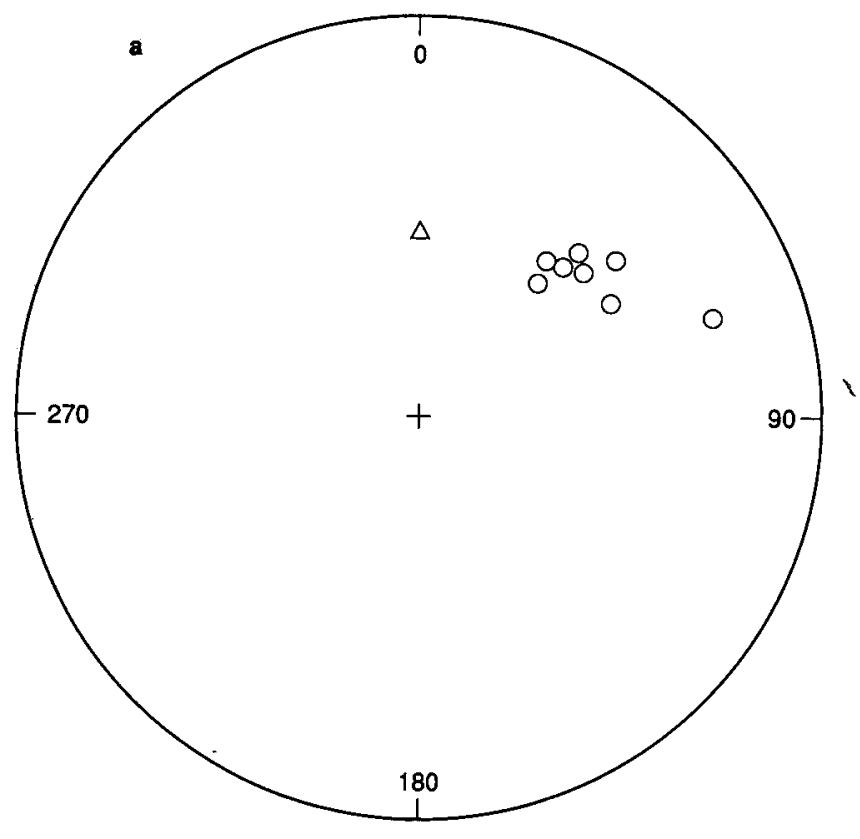

Discussion

This study presents paleomagnetic results from rocks ranging in magnetization age from early Triassic to (probably) early Tertiary, distributed over a relatively large region. Directions within the La Ternera and Quebrada Monardes formations are substantially.uniform throughout the area (lateral dimensions of the order of $50 \mathrm{~km})$, implying the absence of small, variably rotated crustal blocks. For this reason we have calculated poles for the La Ternera and Quebrada Monardes formations by combining all respective site mean VGP (bottom of Tables 1 and 2). Comparison of poles from the La Ternera and Quebrada Monardes formations (tilt corrected) with their respective references poles points to about $25^{\circ}$ of clockwise rotation, with no significant latitudinal transport (Table 6). Within the Cerrillos CEG Formation only R polarity is present, and the observed increase in dispersion when the tilt correction is made suggests remagnetization. Furthermore, magnetizations within the cerrillos CEG sites are complicated and difficult to isolate. Another complication in interpreting the Cerrillos CEG results is that we are unsure of the correct reference pole, as explained earlier. These uncertainties inevitably make any tectonic conclusions tentative. The rotation listed in Table 6 for Cerrillos CEG $\left(17.3^{\circ}\right)$ is only slightly more than half that of the older format tions in the same area.

Cerrillos EDB exposures have only $\mathrm{N}$ polarity. (Thus the two Cerrillos localities do not have the same age of magnetization.) After site 90 eb70 is dropped, scatter is improbably low (Table 3 ), possibly because remagnetization occurred so rapidly that the secular variation was not fully sampled. Thus the Cerrillos EDB direction is doubly suspect. However, for what it is worth, it shows an unusually large clockwise rotation $\left(48.1^{\circ}\right)$, with insignificant latitudinal displacement.

The essentially identical rotation found in upper Triassic and Upper Jurassic rocks from the eastern sampling area suggests that rotation did not begin until sometime after the late Jurassic. The fact that the cerrillos CEG locality has a (poorly defined) rotation of about $15^{\circ}$ may mean that rotation was roughly half complete when these rocks were remagnetized, presumably in the early Tertiary.

Results from the Cerrillos EDB locality are interesting, although unfortunately not particularly reliable. Cerrillos EDB rocks apparently were thoroughly remagnetized, at some time when the geomagnetic field had normal polarity; time of remagnetization is otherwise poorly constrained but probably was early Tertiary. The tight cluster of site mean directions for the EDB locality suggests that the secular variation

Fig. 7. Cerrillos Formations, Eliza del Bordo has not been completely averaged, and the fact

TABLE 5. Reference Poles

\begin{tabular}{|c|c|c|c|c|}
\hline Time Interval & South Latitude & East Longitude & $A_{95}$ & Reference \\
\hline Iriassic & $82.7^{\circ}$ & $285.5^{\circ}$ & 7.4 & $\operatorname{TC} 1-5$ \\
\hline Jurassic & $89.1^{\circ}$ & $217.1^{\circ}$ & 4.6 & Beck [1988] \\
\hline Early Tertiary & $85.1^{\circ}$ & $303.2^{\circ}$ & 10.0 & $D$ and $E$ \\
\hline
\end{tabular}

References are TC1, Veldcamp et al. [1971]; TC2, sum of Thompson [1972], Valencio et al. [1977], and Creer et al. (1970]; TC3, Valencio [1970]; TC4, Valencio et al. [1975]; and TC5, sum of Valencio et al. [1975], Creer et al. [1970]. D and $E$ reference indicates early Tertiary North American reference pole of Diehl et al. [1983] rotated into South American reference frame using reconstruction parameters of Engebretson et al. [1985]. A95 is radius of circle of $95 \%$ confidence. Circle of confidence for early Tertiary pole is assigned arbitrarily. See text. 
TABLE 6. Displacements of the Various Sampling Areas Relative to stable South American, Calculated From Paleomagnetic Poles

\begin{tabular}{|c|c|c|c|c|c|c|}
\hline & \multicolumn{2}{|c|}{ Observed } & \multicolumn{2}{|c|}{ Reference } & \multirow[b]{2}{*}{ Rotation } & \multirow[b]{2}{*}{$\begin{array}{l}\text { Poleward } \\
\text { Transport }\end{array}$} \\
\hline Formation & Pole & $\alpha 95$ & Pole & $\alpha 95$ & & \\
\hline
\end{tabular}

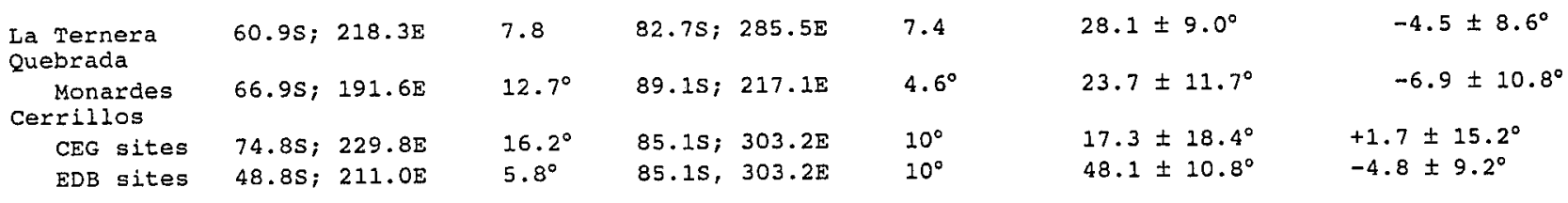

Observed poles are mean virtual geomagnetic poles, from Tables 1-3. Reference poles are summarized in Table 5 and discussed in text. Rotation and poleward transport were calculated using poles from Beck [1989]. positive rotations are clockwise; positive poleward transport is northward.

that the rocks are remagnetized opens the possibility of post magnetization tilting. If neither of these problems is substantial, then the Cerrillos EDB locality has a large rotation, perhaps as much as $45^{\circ}$. This would indicate that the EDB and CEG localities have different rotational histories and therefore are located in different structural blocks. This tentative interpretation obviously needs to be tested by further work.

vinally, with respect to the ultimate cause of rotation, it is worth noting that our results do not match the predictions of the orocline model (Figure la) of Isacks [1988] particularly well. Isacks model calls for about $10^{\circ}-15^{\circ}$ of clockwise rotation on the southern limb of the orocline, far less than we encountered. Furthermore, Isacks envisaged most of the rotation as occurring in a single episode, during the Miocene. Our results (excluding the cerrillos EDB locality) are more compatible with mechanisms that produce time-dependent rotation, with some of the rotation having been acquired during the Mesozoic. However, results from north of Arica match the predictions of the Isacks [1988] model (P. D. Riley, unpublished compilation, 1992). It seems very likely that results south of the Arica deflection reflect both mechanisms: rotations of small blocks in response to oblique subduction added to the effects of oroclinal bending.

\section{Acknowledgments. We thank wilfredo} Pincheira, Antonio Dias Tapia, Jose Cembrano, Robert Drake, and many geologists of the Servicio Nacional de Geologia y Mineria, Chile, for help in the field. This study was funded by NSF Grant EAR 8718896, National Geographic Society grant 3738-88, and a student research grant to P.D.R. from Sigma Xi. The North American authors are grateful to the Servicio Nacional de Geologia y Mineria, Chile, for logistical support. Reviews by R. Butler and J. Irwin were useful. The authors particularly thank A. Griscom for tirelessly and meticulously checking our logic, syntax, grammar and even our spelling.

\section{References}

Beck, M.E., Jr., Discordant paleomagnetic pole positions as evidence of regional shear in the western Cordillera of North America, Am. J. Sci., 276, 694-712, 1976.

Beck, M.E., Jr., Tectonic rotations on the leading edge of South America: The Bolivian orocline revisited, Geology, 15, 806-808, 1987.

Beck, M.E., Jr., Analysis of Late JurassicRecent paleomagnetic data from active plate margins of South America, J. South Am. Earth Sci., $1,39-52,1988$.

Beck, M.E., Jr., Paleomagnetism of continental North America; Implications for displacement of crustal blocks within the Western cordillera, Baja California to British Columbia, in Geophysical Framework of the Continental United States, edited by L. Pakiser and W. Mooney, Mem. Geol. Soc. Am., 172, 471-492, 1989 .

Beck, M.E., Jr., R.F. Burmester, D.E. Craig, C.S. Gromme, and R.E. Wells, Paleomagnetism of Middle Tertiary volcanic rocks from the Western Cascade Series, Northern California, J. Geophys. Res., 91, 8219-8230, 1986.

Bell, C., the lower Paleozoic metasedimentary basement of the Coastal Range of chile, between $28^{\circ} 30^{\prime}$ and $27^{\circ} \mathrm{S}$, Rev. Geol. Chile, $17,21-29,1982$.

Bellieni, G., P. Brotzu, P. Comin-Chiaromonti, M. Ernesto, A. Melfi, I. Pacca, F. Piccirillo, and D. Stolfa, Petrological and paleomagnetic data on the plateau basalt and rhyolite sequences of southern Parana basin (Brazil), An. Acad. Brasil. Cienc., 55, 355383,1983 .

Butler, R.F., F. Hervé, F. Munizaga, M. Beck, R. Burmester, and E. Oviedo, Paleomagnetism of the Patagonian plateau basalts, southern Chile and Argentina, J. Geophys. Res., 96, 6023-6034, 1991 .

Carey, S.W.. The orocline concept in geotectonics, Proc. Soc. Tasmania, 89, 255-288, 1954.

Cox, A., Confidence limits for the precision parameter k, Geophys. J. R. Astron. Soc., 17, 545-549, 1969 .

Creer, K., B. Embleton, and D. Valencio, Triassic and Permo-Triassic paleomagnetic data from South America, Earth Planet. Sci. Lett. 8, 173-178, 1970 .

Diehl, J.F., M.E. Beck, Jr., S. Beske-Diehl, D. Jacobson, and B.C. Hearn, Jr., Paleomagnetism of the Late Cretaceous-Early Tertiary north-central Montana alkalic province, $\underline{\mathrm{J}}$. Geophys. Res. 88, 10, 593-10, 609, 1983.

Engebretson, D.C., A. Cox, and R. Gordon, Relative motions between oceanic and continental plates in the Pacific basin, Spec. Pap. Geol. Soc. Am. 206, 59 pp., 1985.

England, P., and R.E. Wells, Neogene rotations and quasicontinuous deformation of the Pacific Northwest continental margin, Geology, 19, 978-981, 1991.

Fisher, R.A., Dispersion on a sphere, Proc. R. Soc. London Ser. A, 217, 295-305, 1953.

Hartley, A.J., P. Turner, G. Williams, and s. Flint, Paleomagnetism of the Cordillera de la Costa, northern Chile: Evidence for local forearc rotation, Earth Planet. Sci. Lett.e 
89, 375-386, 1988.

Irving, E., and G.A. Irving, Apparent polar wander paths, Carboniferous through Cenozoic, and the assembly of Gondwana, Geophys. Surv. $5,141-188,1982$.

Irving, E., J. Monger, and R. Yole, New paleomagnetic evidence for displaced terranes in British Columbia, in The Continental Crust and Its Mineral Deposits, edited by $D$. Strangway, Geol. Assoc. Can. Spec. Pap. 20, $441-456,1980$.

Irwin, J., W. Sharp, R. Spangler, and R. Drake, Some paleomagnetic constraints on the tectonic evolution of the Coastal cordillera of central Chile, J. Geophys. Res., 92, 3603$3614,1987$.

Isacks, B., Uplift of the central Andes plateau and bending of the Bolivian orocline, $\mathrm{J}$. Geophys. Res.. 93, 3211-3231, 1988.

Kirschvink, J., The least-squares line and plane and the analysis of paleomagnetic data, Geophys. J.R. Astron. Soc., 62, 699-718, 1980 .

Kono, M., Y. Fukao, and A. Yamamoto, Mountain builaing in the central Andes, J.Geophys. Res. 94, 3891-3905, 1989.

Mpodozis, C., and V. Ramos, The Andes of Chile and Argentina, in Geology of the Andes and Its Relation to Hydrocarbon and Mineral Resources, edited by G. Ericksen, M. CanaPinochet, and J. Reinemund, pp. 59-90, Circum-Pacific Council for Energy and Mineral Resources, Houston, Tex., 1990.

Ramos, V., T. Jordon, R. Allmendinger, C. Mpodozis, S. Kay, J. Cortes, and M. Palma, Paleozoic terranes of the Central Andes, Tectonics, 5, 855-880, 1986.

Sepulveda, P., and J. Naranjo, Hoja Carrera Pinto, Region de Atacama, Santiago, Chile, Carta Geol. de Chile 53, 62 pp., Ser. Nac. de Geol. Y Miner., Santiago, 1982.

stone, D., B. Panuska, and D. Packer, Paleolatitudes versus time for southern Alaska, J. Geophys. Res., 87, 3697-3707, 1982.
Thompson, R., Paleomagnetic results from the Paganzo basin of northwest Argentina, Earth Planet. Sci. Lett. . 27, 145-156, 1972.

Valencio, D., El paleomagnetismo de una formacion magmatica del suroeste de la Provincia de Mendoza, de Cuartas J. Geol. Argentina, 20, 191-198, 1970 .

Valencio, D., J. Mendia, and J. Vilas, Paleomagnetism and $\mathrm{K}-\mathrm{Ar}$ ages of Triassic igneous rocks from the Ischigualasto-Ischichuca basin and Puesto Viejo formation, Argentina, Earth Planet Sci. Lett., 26, 319-330, 1975.

Valencio, D., J. Vilas, and J. Mendia, Paleomagnetism of a sequence of redbeds of the middle and upper sections of Paganzo Group (Argentina), and the correlation of Upper Paleozoic-Lower Mesozoic rocks, Geophys. J.R. Astron. Soc., 51, 47-58, 1977 .

Veldkamp, J., F. Mulder, and J. Zijderveld, Paleomagnetism of Surinam dolerites, Phys. Earth Planet. Inter., 4, 370-380, 1971 .

zentilli, M., Geological evolution and metallogenetic relationship in the Andes of northern Chile between $26^{\circ}$ and $29^{\circ} \mathrm{S}, \mathrm{Ph} . \mathrm{D}$. dissertation, 446 pp., Queens Univ. location, Kingston, Ontario, Canada, 1974.

M.E. Beck, Jr., R.F. Burmester, and P.D. Riley, Department of Geology, Western Washington University, Bellingham, WA 98225.

A. Garcia, Geoestudios, Ltda., Genova 209s, Santiago, Chile.

c. Mpodozis, Servicio Nacional de Geologia y Mineria-Chile, Ave. Santa Maria 0104, Santiago, Chile.

(Received March 26, 1992;

revised October 19, 1992;

accepted November $12,1992$. ) 